\title{
Functional connectivity changes of primary somatosensory cortex in response to acupuncturing in bell's palsy patients
}

\begin{abstract}
Bell's palsy (BP), an idiopathic and unilateral facial paralysis, is frequently treated by acupuncture in Asia. This study aims to investigate the connectivity changes of the primary somatosensory cortex (SI) induced by acupuncturing at ipsilateral and contralateral Hegu acupoints of BP patients. Fifteen healthy volunteers and thirty BP patients were recruited, who underwent the resting-state functional MRI scanning before and after acupuncture. All subjects received acupuncture treatment at either ipsilateral or contralateral Hegu acupoint. Functional connectivity of bilateral SI was calculated for further intergroup analysis. The results showed significant functional connectivity alterations in the contralateral acupoint group $(\mathrm{P}<0.01)$ while no significant alterations were observed in neither the ipsilateral acupoint groups nor the healthy control groups. For the SI connectivity alteration, a significant difference was observed between the contralateral group and the ipsilateral group $(\mathrm{P}<0.01)$. These results suggested that acupuncturing at the contralateral and ipsilateral Hegu acupoint could induce different alterations in functional connectivity in recovery from BP. Therefore, the recovery mechanism of acupuncturing might vary from side to side.
\end{abstract}

Keywords: bell's palsy, functional mri, primary somatosensory cortex, compensatory reaction, functional connectivity
Volume 5 Issue 5 - 2017

\author{
Jiali Xu,' Bohan Liu, ${ }^{2}$ Chunsheng Xu, ${ }^{1,2,3}$ \\ Sheng Hu,' Yifang Zhu, ${ }^{2}$ Hongxing Kan, ${ }^{3}$ \\ Bensheng Qiu, ${ }^{1,3, \#}$ Chuanfu Li, ${ }^{2}$ Jun Yang ${ }^{1,2}$ \\ \#Both authors are Equal contribution \\ 'Centers for Biomedical Engineering, University of Science and \\ Technology of China, China \\ ${ }^{2}$ Laboratory of Digital Medical Imaging, The First Affiliated \\ Hospital of Anhui University of TCM, China \\ ${ }^{3}$ Anhui Computer Application Institute of Traditional Chinese \\ Medicine, China
}

\begin{abstract}
Correspondence: Bensheng Qiu, Centers for Biomedical Engineering, University of Science and Technology of China, Hefei, Anhui 230027, China, Tel 86-55I-63600583
\end{abstract} Email bqiu@ustc.educn

Co-correspondence: Jun Yang, Laboratory of Digital Medical Imaging, The First Affiliated Hospital of Anhui University of TCM, Hefei, Anhui 23003I, China

\#Both authors are Equal contribution

Received: November 28, 2017 | Published: February 21, 2017
Abbreviations: SI, primary somatosensory cortex ; FMRI, functional magnetic resonance imaging; BP, bell's palsy; HBS, house-brackman facial nerve grading system; SFG, superior frontal gyrus; STG, superior temporal gyrus; MTG, middle temporal gyrus; MFG, middle frontal gyrus; ACC, anterior cingulate cortex; PCC, posterior cingulate cortex; MI, primary motor cortex; IFG, inferior frontal gyrus; SPL, superior parietal lobule; MOG, middle occipital gyrus; MCC, middle cingulate cortex; MFG, middle frontal gyr.us; SII, secondary somatosensory cortex

\section{Introduction}

Unilateral peripheral facial palsy (PFP), known as idiopathic facial paralysis or Bell's palsy (BP), is a common facial nerve-lesioned disease characterized with abnormal facial features and uncontrolled facial muscle activities. The basic pathogenesis of BP is that the impaired efferent nerve leads to the inability of performing facial movements. Acupuncture as an important component of Chinese medical system is frequently used to treat Bell's palsy by inserting the fine needles into specific acupoints. ${ }^{1}$ Although the cellular and molecular constituents of this therapy remain largely unknown, the curative effect of acupuncture treatment on BP is verified experimentally and clinically. ${ }^{2}$ Prevailing theories suggest that the effects of acupuncture stimulation are focused on the change of brain functional network. ${ }^{3}$

Hegu acupoint (LI4), one of the primary acupoints on the back of the hand, is often used for acupuncture treatment of BP clinically. Acupuncturing the acupoint would directly stimulate the somatoreceptors on the skin and the impulse would be transmitted to the intermediolateral cell column via the sympathetic nerve fibers. Finally, the impulse would be transmitted to the cerebral cortex and lead to cerebral activation. ${ }^{4}$ A possible consideration of choosing Hegu for treating BP is that the representative cortical areas of the face and hands are adjacent in the primary motor area (MI) and the primary somatosensory area (SI). ${ }^{5}$ In BP patients, the cortical reacting field of hands extends laterally and enlarges into the site of facial regions in SI. ${ }^{6}$ At present, Hegu acupoint on the contralateral side of the palsy is selected as the primary acupoint in clinical practice, and the ipsilateral Hegu point is selected as the secondary acupoint. This is because, according the clinical results, acupuncturing at the contralateral Hegu point is more effective. Questions have been raised on whether contralateral acupuncture would induce different curative effect from ipsilateral acupuncture. According to recent reports on cortical reorganization of recovery from $\mathrm{BP},{ }^{7,8}$ we presumed that acupuncturing at the contralateral side was more effective in brain functional reorganization.

To test this hypothesis, this study was designed to examine the possible mechanism of acupuncture treatment for Bell's palsy. As is known, brain is capable of adapting to new situations by cortical plasticity and functional reorganization, which can be reflected by the changes of functional connectivity. Our previous studies have also shown that the acupuncture leads to significant connectivity change. In addition, several investigations suggest the brain's responses to acupuncture depend on the functional connectivity status and that some functional reorganizations of special cortical regions are important in the process of BP recovery. ${ }^{5,9,10}$ Thus, we believed that different effects of acupuncture made on the bilateral Hegu points 
would be revealed by comparing functional connectivity alterations. Correspondingly, the reason of choosing SI as the region of interest (ROI) is that SI is consistently activated in response to acupuncture at Hegu point and that SI connectivity is related to the brain status in BP patients. ${ }^{11}$ Therefore, in this study, we sought to explore SI connectivity changes in response to contralateral and ipsilateral acupuncture at Hegu acupoints using functional MRI.

\section{Materials and methods}

\section{Subjects}

In this study, the disease duration and the House-Brackman facial nerve grading system (HBS; $1=$ normal facial movement, $6=$ no movement, scored by an experienced acupuncturist with no prior knowledge on the data results) were introduced to estimate patients condition. 30 patients with unilateral facial palsy (13 women and 17 men, aged from 19 to 46 years duration data $<14$ days, HBS $>3$ ) were recruited in this study. All of them were right-handed with no pregnancy, psychiatric diseases, neurological or systemic diseases, or other factors that might take effects on this study. To extract ROIs, 15 healthy right-handed subjects ( 8 women and 7 men, aged from 18 to 29years) were recruited in the hospital. These healthy volunteers were acupuncture naïve and were diagnosed with no congenital diseases, immune and endocrine disorders, or neuropsychiatric diseases. All patients were divided into two groups randomly: the ipsilateral acupoint group (6 right-side palsy, 9 left-side palsy) and the contralateral acupoint group ( 7 right-side palsy, 8 left-side palsy). In the ipsilateral group, acupuncture was operated at the Hegu on the facial palsy side, while on the opposite side in the contralateral group. Participants gave written informed consent and all study protocols were approved by the human research committee of the First Affiliated Hospital of Anhui University of Chinese Medicine.

\section{Experimental design}

All subjects in the two study groups were required to take resting-state fMRI scanning before the acupuncture and the first data was acquired with collecting 200time points for $10 \mathrm{~min}$. Then, an acupuncture needle was inserted into either contralateral or ipsilateral Hegu acupoint. When the de-qi sensation was acquired, a second fMRI data was acquired with collecting 200time points for $10 \mathrm{~min}$. Finally, the needle was pulled out and the third data was acquired with collecting 200 time points for $10 \mathrm{~min}$.

To define the seed regions of SI, task-state fMRI data of 15 healthy volunteers were acquired. During the task-state fMRI scans, a professional acupuncturist was requested to retain and rotate needles at Hegu points alternately. 160time points were collected during the task-state fMRI session for 10 minutes and 40 seconds, including 32time points of resting-state, 32time points of task-state of rotating needle, 48time points of resting-state, 32time points of task-state of rotating needle, and 16time points of resting-state.

\section{Image acquisition}

During the acupuncture and fMRI, all the participants were required to lie supine with ear plugs and eyes closed so that unnecessary visual stimulation that could lead to sleep or distraction were minimized. The fMRI data acquisition was performed on a Siemens Symphony 1.5T MRI whole-body scanner (Siemens Healthcare, Germany) with a standard head coil. The MR imaging protocol included: 1) pilot imaging; 2) T2-weighted imaging to eliminate any apparent diseases in the brains; 3) two-dimensional (2D) T1-weighted imaging, covering the entire brain with a total of 36 whole-brain slices, using a spoiled gradient echo sequence $\left(\mathrm{TR} / \mathrm{TE} / \mathrm{FA}=500 \mathrm{~ms} / 12 \mathrm{~ms} / 90^{\circ}\right.$, field of view $($ FOV $)=230 \mathrm{~mm}^{\prime} 230 \mathrm{~mm}$, slice $\left./ \mathrm{gap}=3.0 \mathrm{~mm} / 0.75 \mathrm{~mm}\right)$ and a matrix of $192^{\prime} 144$; 4) resting-state fMRI imaging before acupuncture to obtain the blood oxygenation level dependent (BOLD) signal, covering the entire brain with a total of 36 whole-brain slices, using an EPI-BOLD sequence $\left(\mathrm{TR} / \mathrm{TE} / \mathrm{FA}=3000 \mathrm{~ms} / 30 \mathrm{~ms} / 90^{\circ}, \mathrm{FOV}=192 \mathrm{~mm}^{\prime} 192 \mathrm{~mm}\right.$, slice $/$ gap $=3.0 \mathrm{~mm} / 0.75 \mathrm{~mm}$ ) and a matrix of $64^{\prime} 64 ; 5$ ) restingstate fMRI imaging during acupuncture with the same parameters; 6) resting-state fMRI imaging after acupuncture with the same parameters; 7) task-state fMRI imaging, covering the entire brain with a total of 36 whole-brain slices, using an EPI-BOLD sequence $\left(\mathrm{TR} / \mathrm{TE} / \mathrm{FA}=4000 \mathrm{~ms} / 50 \mathrm{~ms} / 90^{\circ}, \mathrm{FOV}=192 \mathrm{~mm}{ }^{\prime} 192 \mathrm{~mm}\right.$, slice $/$ gap $=3.0 \mathrm{~mm} / 0.75 \mathrm{~mm}$ ) and a matrix of $\left.64^{\prime} 64 ; 8\right)$ three-dimensional (3D) T1-weighted anatomical sagittal imaging, covering the whole-brain with a total of 176 slices, using a spoiled gradient echo sequence $\left(\mathrm{TR} / \mathrm{TE} / \mathrm{FA}=2100 \mathrm{~ms} / 3.93 \mathrm{~ms} / 13^{\circ}, \mathrm{FOV}=250 \mathrm{~mm}^{\prime} 250 \mathrm{~mm}\right.$, slice $/$ gap $=1.0 \mathrm{~mm} / 0.5 \mathrm{~mm}$ ) and a matrix of $256^{\prime} 256$. In this study, the 7 th image sequence was used to define seed ROIs, and the data acquired from the 4 th and 6 th image sequences were applied in further processing.

\section{fMRI data preprocessing}

The preprocessing of all fMRI data was done by the combination of three softwares, FSL (FMRIB's Software Library, Oxford, UK), AFNI (Analysis of Functional Neuro Images, Medical College of Wisconsin, USA), and FreeSurfer (Athinoula A. Martinos Center for Biomedical Imaging, Massachusetts General Hospital, USA). Firstly, a unified matrix was utilized to reconstruct and realign the anatomical and functional images. Then, after performing the skull stripping and head motion correction, the co-registration from the functional images to the anatomical images and the normalization to Montreal Neurological Institute 152 (MNI152) standard brain were applied. Afterwards, to reduce low-frequency drifts and highfrequency noises, all of these fMRI data were filtered by a band-pass filter $(0.007 \mathrm{~Hz}$ to $0.1 \mathrm{~Hz})$ and smoothed by a $5-\mathrm{mm}$ full-width at half maximum Gaussian kernel. After preprocessing, the individual fourdimensional data were acquired for group analyses.

\section{Extraction of region of interest}

Statistical activation maps were completed via a generalized linear model (GLM) respectively. Based on these maps, the seed regions with 33 voxels (voxel size $2 \times 2 \times 2 \mathrm{~mm}$ ) and diameter of $4 \mathrm{~mm}$ were extracted from the hand field in the right SI of healthy participants. The seed region of left SI was extracted from task-state fMRI in a similar manner.

\section{Functional connectivity analysis}

After the ROI were extracted, the functional connectivity of bilateral SI seed regions was calculated as follows. First, the time courses of cerebrospinal fluid, cerebral white matter, seed regions of bilateral SI were extracted. Second, several sources of variation were removed by using the linear regression model: 1) six parameters of rigid head motion correction; 2) the signal of cerebrospinal fluid; 3) the signal from cerebral white matter. A generalized linear model was also carried out to calculate the individual statistical maps.

\section{Group analysis}

The image data of patients with left-side facial palsy were mirror flipped across the midsagittal plane before the group analyses so that the analyses were based on the data of single-side facial palsy. Then, group analysis was performed by the paired t-test between before 
and after acupuncture mappings. Thereafter, bilateral SI regions were extracted to compute the functional connectivity for all groups.

In order to eliminate the individual variation, four group data (before and after acupuncture mappings in two study groups) were included in statistical design for intergroup t-test between contralateral group and ipsilateral group, i.e. (CON_after - CON_before) - (IPS after - IPS before)..$^{12}$ The results were corrected by the Monte Carlo method, with $P$ value of $0.01, \alpha$-value of 0.02 , and cluster size of 134 voxels.

\section{Results}

\section{The healthy control group}

No significant alteration in bilateral SI connectivity was observed

\section{The ipsilateral acupoint group}

No significant alteration in bilateral SI connectivity was observed.

\section{The contralateral acupoint group}

A significant decrease in right SIconnectivity was observed in bilateral superior frontal gyrus (SFG), superior temporal gyrus (STG), right SI, middle temporal gyrus (MTG), middle frontal gyrus (MFG), anterior cingulate cortex (ACC), posterior cingulate cortex (PCC), precuneus, left primary motor cortex (MI), inferior frontal gyrus (IFG), superior parietal lobule (SPL), cuneus (Figures 1 \& Table 1). For left SI connectivity, a significant decrease was observed in bilateral middle occipital gyrus (MOG), IFG, STG, right middle cingulate cortex (MCC), middle frontal gyrus (MFG), and left MI, MTG (Figures $2 \&$ Table 2).

\section{The intergroup analysis}

A significant difference of alterations in right SI connectivity between two study groups was observed in right PCC and right precuneus (Figures $3 \&$ Table 3). For the alterations in left SI connectivity, the difference was observed in right MTG, secondary somatosensory cortex (SII), ACC, left MTG, cuneus (Figures 4 \& Table 4).

Table I Group analysis results of areas with altered connectivity with right SI in the contralateral acupoint group

\begin{tabular}{llllll}
\hline MNI coordinates & & & & & \\
region (BA) & Side & $\mathbf{X}(\mathbf{m m})$ & $\mathbf{Y}(\mathbf{m m})$ & $\mathbf{Z}(\mathbf{m m})$ & Peak (Z value) \\
\hline Superior frontal gyrus, SFG & $\mathrm{L}$ & -22 & 64 & 8 & -4.34 \\
Superior frontal gyrus, SFG & $\mathrm{L}$ & -12 & 10 & 58 & -3.80 \\
Inferior frontal gyrus, IFG & $\mathrm{L}$ & -48 & 26 & -8 & -3.59 \\
Superior temporal gyrus, STG (22) & $\mathrm{L}$ & -48 & -60 & 12 & -3.75 \\
Precentral gyrus, MI (4) & $\mathrm{L}$ & -42 & -18 & 38 & -4.16 \\
Superior parietal lobule, SPL (7) & $\mathrm{L}$ & -22 & -62 & 44 & -3.98 \\
Cuneus & $\mathrm{L}$ & -18 & -78 & 14 & -4.07 \\
Precuneus & $\mathrm{R}$ & 38 & -72 & 34 & -3.99 \\
Anterior cingulate cortex, ACC & $\mathrm{R}$ & 10 & 20 & 30 & -4.17 \\
Posterior cingulate cortex, PCC & $\mathrm{R}$ & 18 & -54 & 4 & -4.76 \\
Superior temporal gyrus, STG & $\mathrm{R}$ & 66 & -30 & 6 & -4.80 \\
Middle frontal gyrus, MFG (6) & $\mathrm{R}$ & 32 & 4 & 56 & -3.99 \\
Postcentral gyrus, SI(3) & $\mathrm{R}$ & 44 & -24 & 64 & -4.12 \\
Middle temporal gyrus, MTG & $\mathrm{R}$ & 62 & -18 & -16 & -4.15 \\
Superior frontal gyrus, SFG & $\mathrm{R}$ & 20 & 44 & -16 & -4.36
\end{tabular}

Table 2 Group analysis results of areas with altered connectivity with left SI in the contralateral acupoint group

\begin{tabular}{llllll}
\hline $\begin{array}{l}\text { MNI coordinates } \\
\text { region (BA) }\end{array}$ & Side & $\mathbf{X}(\mathbf{m m})$ & $\mathbf{Y}(\mathbf{m m})$ & $\mathbf{Z}(\mathbf{m m})$ & Peak $(\mathbf{Z}$ Value) \\
\hline Precentral gyrus, MI & $\mathrm{L}$ & -44 & -6 & 28 & -4.15 \\
Inferior frontal gyrus, IFG & $\mathrm{L}$ & -24 & 24 & -10 & -4.07 \\
Middle temporal gyrus, MTG & $\mathrm{L}$ & -68 & -24 & -16 & -3.69 \\
Superior temporal gyrus, STG (22) & $\mathrm{L}$ & -42 & -20 & -6 & -3.67 \\
Middle occipital gyrus, MOG (19) & $\mathrm{L}$ & -28 & -86 & 16 & -4.42 \\
Middle occipital gyrus, MOG (19) & $\mathrm{R}$ & 44 & -82 & 4 & -4.49 \\
Middle frontal gyrus, MFG & $\mathrm{R}$ & 40 & 26 & 32 & -4.36 \\
Inferior frontal gyrus, IFG & $\mathrm{R}$ & 46 & 12 & 14 & -3.50 \\
Superior temporal gyrus, STG & $\mathrm{R}$ & 42 & -50 & 8 & -4.46 \\
Middle cingulate cortex, MCC & $\mathrm{R}$ & $\mathrm{I}$ & -38 & 40 & -4.65 \\
\hline
\end{tabular}

Table 3 Intergroup analysis results of areas with altered connectivity with right SI between contralateral acupoint group and ipsilateral acupoint group

\begin{tabular}{llllll}
\hline MNI coordinates & & & & & \\
Region (BA) & Side & $\mathbf{X}(\mathbf{m m})$ & $\mathbf{Y}(\mathbf{m m})$ & $\mathbf{Z}(\mathbf{m m})$ & Peak (Z Value) \\
\hline Posterior cingulate cortex, PCC & $\mathrm{R}$ & 6 & -56 & 20 & -3.91 \\
Precuneus (39) & $\mathrm{R}$ & 42 & -72 & 36 & -4.41 \\
\hline
\end{tabular}


Table 4 Intergroup analysis results of areas with altered connectivity with left SI between contralateral acupoint group and ipsilateral acupoint group

\begin{tabular}{|c|c|c|c|c|c|}
\hline \multicolumn{6}{|l|}{ MNI coordinates } \\
\hline region $(\mathrm{BA})$ & Side & $\mathbf{X}(\mathbf{m m})$ & $\mathbf{Y}(\mathbf{m m})$ & $\mathbf{Z}(\mathbf{m m})$ & Peak (Z Value) \\
\hline Middle temporal gyrus, MTG & $\mathrm{L}$ & -46 & -26 & -8 & -4.06 \\
\hline Cuneus (18) & $\mathrm{L}$ & -16 & -84 & 26 & -3.88 \\
\hline Middle temporal gyrus, MTG & $\mathrm{R}$ & 60 & -44 & -10 & -4.06 \\
\hline Middle temporal gyrus, MTG & $\mathrm{R}$ & 44 & -48 & 6 & -3.79 \\
\hline Inferior parietal lobule, SII & $\mathrm{R}$ & 26 & -44 & 54 & -4.25 \\
\hline Anterior cingulate cortex, ACC (24) & $\mathrm{R}$ & 12 & 2 & 42 & -4.05 \\
\hline
\end{tabular}

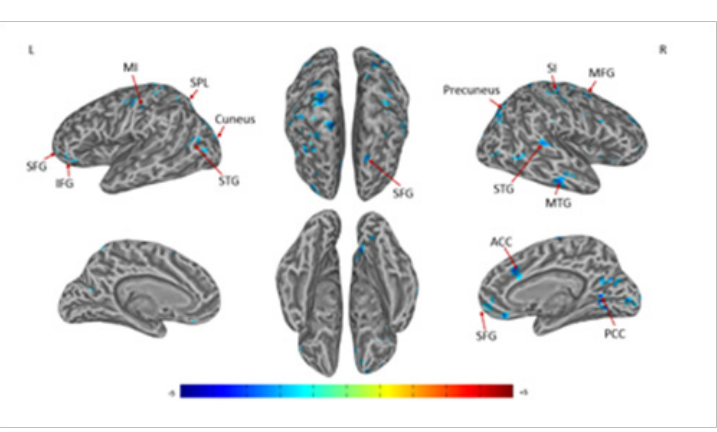

Figure I Connectivity-altered areas with right SI in the contralateral acupoint group. $\mathrm{P} \leq 0.05, \alpha \leq 0.02$, corrected by the Monte Carlo method.

MI: primary motor cortex (precentral gyrus); MFG: middle frontal gyrus; IFG: inferior frontal gyrus; MTG: middle temporal gyrus; SPL: superior parietal lobule; SFG: superior frontal gyrus; SI: primary somatosensory cortex; STG: superior temporal gyrus; PCC: posterior cingulate cortex; ACC: anterior cingulate cortex

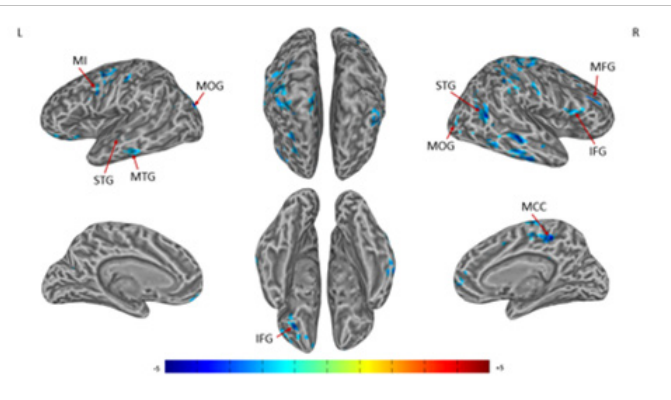

Figure 2 Connectivity-altered areas with left $\mathrm{SI}$ in the contralateral acupoint group. $\mathrm{P} \leq 0.01, \alpha \leq 0.02$, corrected by the Monte Carlo method.

MI, precentral gyrus; MOG, middle occipital gyrus; MFG, middle frontal gyrus; IFG, inferior frontal gyrus; MTG, middle temporal gyrus; STG, superior temporal gyrus; MCC, middle cingulate cortex

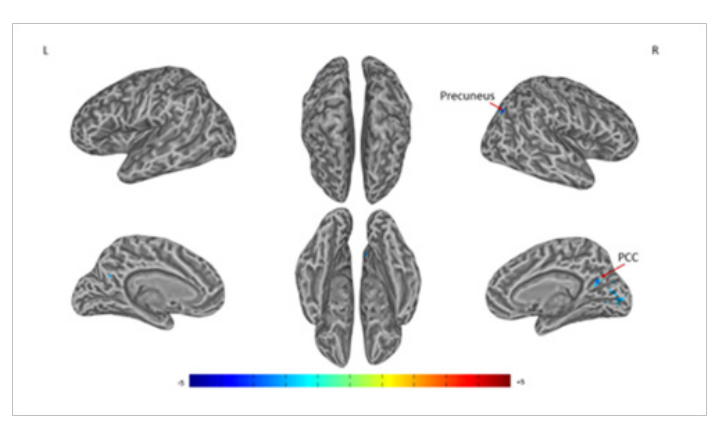

Figure 3 Connectivity-altered areas with right $\mathrm{SI}$ in intergroup analysis.

$\mathrm{P} \leq 0.0 \mathrm{I}, \alpha \leq 0.02$, corrected by the Monte Carlo method. PCC, posterior cingulate cortex

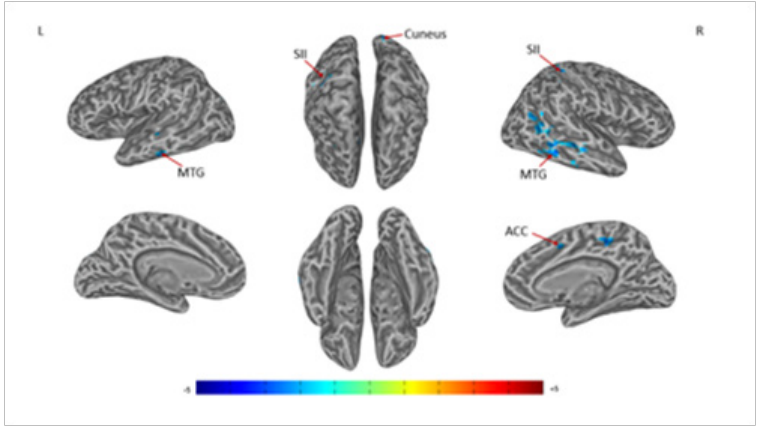

Figure 4 Connectivity-altered areas with left SI in intergroup analysis.

MTG, middle temporal gyrus; SII, secondary somatosensory cortex; ACC, anterior cingulate cortex

Notes of Abbreviations: BA: Brodmann Area; L: Left; R: Right; MNI: Montreal Neurological Institute; MI: Primary Motor Cortex; SI: Primary Somatosensory Cortex The threshold was set to $\mathrm{P} \leq 0.01$, $\alpha \leq 0.02$ (corrected using the Monte Carlo method).

Notes of Abbreviations: BA: Brodmann Area; L: Left; R: Right; MNI: Montreal Neurological Institute; MI: Primary Motor Cortex The threshold was set to $\mathrm{P} \leq 0.01, \alpha \leq 0.02$ (corrected using the Monte Carlo method).

Notes of Abbreviations: BA: Brodmann Area; L: Left; R: Right; MNI: Montreal Neurological Institute; SII: Secondary Somatosensory Cortex The threshold was set to $\mathrm{P} \leq 0.01, \alpha \leq 0.02$ (corrected using the Monte Carlo method).

\section{Discussion}

In this study, we applied fMRI to investigate the difference between contralateral acupuncture and ipsilateral acupuncture. We contrasted their alterations in connectivity and the results showed that the alterations were more obvious in contralateral acupoint group even though the direct activation area of contralateral acupuncture was in the non-lesioned side. It was in accordance with clinical result thatcontralateral acupuncture has stronger therapeutic effect. ${ }^{10,13}$ Our results suggested that acupuncture might improve functions of the lesioned side brain by stimulating non-lesioned side brain.

\section{The possible mechanism of bilateral SI connectivity changes}

Our results showed that the connectivity of non-lesioned side SI was changed by contralateral acupuncture. Therefore, we suggested that the possible mechanism might be relevant to the compensatory reaction of non-lesioned side brain. Normally, the facial representative areas of MI preferentially generate contralateral projections which end up in the facial neurons (efferent nerves) that innervate the lower 
facial musculature. ${ }^{14}$ SI captures the sensory signals of musculature motion through the afferent nerves. Thus, MI, facial musculature and SI form a motor-sensory circuit. In BP patients, the circuit was broken due to the impairments of facial nerves. This signals the brain to attempt to correct the impairments by stimulating the contralateral muscle. This continuous compensatory process in non-lesioned side might lead to excessive expression of brain activity. According to some clinical researches, the contralateral blepharospasm in some BP patients mainly resulted from the compensatory hyper-activities in non-paralyzed side. It has been verified that the acupuncture played an important role in ameliorating the blepharospasm in non-lesioned side. ${ }^{15,16}$ We suggested that the alteration in non-lesioned side SI connectivity might be the result of reductions of the abnormal brain activity tendencies ${ }^{5,10}$.

In the contralateral acupoint group, significantly decreased connectivity of lesioned side SI was also observed in several cortical areas. Different from changed connectivity in non-lesioned SI, the possible mechanism underlying this finding might be relevant to the uncrossed dominance. Although most of the nerve fibers show their crossed innervation, there are a few fibers generating ipsilateral innervation. Once the contralateral nerve fibers or the conductive pathways are broken, the ipsilateral innervation becomes activated. ${ }^{17}$ In BP patients, the non-lesioned side cortex tended to show uncrossed innervation and led to the disorder of other brain activities. We suggested that contralateral acupuncture stimulation alleviated this tendency and preserved the crossed innervation. In a word, acupuncture could adjust inaccurate brain autonomic conditioning and provide assistance for the correct cortical reorganization.

\section{Connectivity decreased in specific areas in response to acupuncture}

In this study, a significant decrease in connectivity was observed in the premotor area (SFG, MFG, IFG), temporal lobe (STG, MTG), default mode network (PCC, precuneus), SI, SII, MI, ACC, MCC, cuneus, superior parietal lobule, and middle occipital gyrus. These changes in connectivity could be the result of regulating abnormal brain connectivity and repairing lesioned regions.

Located in the frontal lobe, PMA nearly stays out of the facial motion in healthy individuals. But in BP patients, due to some negative change in motor cortex, PMA would receive projection from facial nerves and directly transmit signals to facial moto-neurons. ${ }^{18}$ Actually, PMA was reorganized as a hub in the facial motor network and became crucial in facial motions. ${ }^{19}$ Therefore, we suggested that an alteration in PMA-SI connectivity could offer help for cortical reorganization of facial motor network.?

The temporal lobe consists of structures that are vital for memories and cognitions. And most of its nerve cells tend to assist in responding to other reactions such as facial expressions..$^{20}$ Indirect evidence in patients with temporal lobe epilepsy shows that the temporal lobe is also associated with facial emotional expression. Thus, we suggested that connectivity changes in temporal lobe could be the result of restoring some facial motions of emotional expressions. ${ }^{18}$

In addition to sensorimotor association areas, changes were also observed in some default mode network (DMN) related areas (PCC, precuneus). DMN is a network relating several brain regions while its major function is to increase the interaction among these regions. ${ }^{21}$ According to a previous study, altering connectivity of DMN-related areas can modulate homeostasis to help recovering from BP. ${ }^{5}$ PCC, located in the backmost part of cingulate cortex, is a key node in DMN. PCC is the representative cortical area of closing eyes movements in some situation like facial palsy. ${ }^{22}$ As a part of the limbic lobe, PCC also plays an important role in the emotional activities. Being adjacent to the posteromedial portion of the parietal lobe, precuneus is responsible for the emotional and conscious adjustment. ${ }^{23}$ Accordingly, we suggested that connectivity changes in some DMNrelated areas could be the result of regulating the emotional disorder which is known as an early symptom of BP patients. ${ }^{24}$

\section{Limitation of this study}

There were several limitations in this study. Firstly, before group analysis, mirror flip was performed. Although the factor of whether the data were flipped was considered in the group analysis, ${ }^{7}$ it also could cause several bias due to the asymmetry of cerebral anatomy and function. Therefore, further studies should control these relevant variables strictly to make the conclusion more convincing.,59

\section{Conclusion}

This study suggested that changes in bilateral SI connectivity induced by the contralateral acupuncture are different from the ipsilateral acupuncture. And the possible mechanism of contralateral acupuncture might be relevant to ameliorating incorrect brain reactions and promoting cortical reorganizations for recovery from BP.

\section{Acknowledgments}

This study was supported by grants from the National Key Basic Research and Development Program (973) under Grant no. 2010CB530500, the National Natural Science Foundation of China under Grant nos. 81202768, Anhui Provincial Natural Science Foundation under Grant no. 1208085MH147, Major Scientific Projects of Anhui Provincial Education Commission under Grant no. KJ2011ZD05, Open Project Program of Institute of Acupuncture and Moxibustion China Academy of Chinese Medical Science under Grant no. ZZKF08010.

J. Xu participated in paper preparation. B. Liu participated in paper preparation. C. Xu participated in paper revision. S. Hu participated in data processing. Y. Zhu participated in data processing. H. Kan participated in data acquisition. C. Li participated in experimental design, data acquisition, data processing, and paper revision. J. Yang and B. Qiu participated in paper revision.

\section{Conflicts of interest}

Author declares there are no conflicts of interest.

\section{Funding}

None.

\section{References}

1. Kwon HJ, Kim JI, Lee MS, et al. Acupuncture for sequelae of Bell's palsy: a randomized controlled trial protocol. Trials. 2011;16:246.

2. Xu SB, Huang B, Zhang CY, et al. Effectiveness of strengthened stimulation during acupuncture for the treatment of Bell palsy: a randomized controlled trial. CMAJ. 2013;185(6):473-479.

3. Dhond RP, Yeh C, Park K, et al. Acupuncture modulates resting state connectivity in default and sensorimotor brain networks. Pain. 2008;136(3):407-418.

4. Dornette WH. The anatomy of acupuncture. Bull N Y Acad Med. 1975;51(8):895-902. 
5. He XX, Zhu YF, Li CF, et al. Acupuncture-induced changes in functional connectivity of the primary somatosensory cortex varied with pathological stages of Bell's palsy. Neuroreport. 2014;25(14):1162-1168.

6. Rijntjes M, Tegenthoff M, Liepert J, et al. Critical reorganization in patients with facial palsy. Annals of neurology. 1997;41:621-630.

7. Klingner CM, Volk GF, Maertin A, et al. Cortical reorganization in Bell's palsy. Restorative neurology and neuroscience. 2011;29(3):203-214.

8. Rijntjes M, Leonhardt G, Jueptner M, et al. Reorganization in the adult human cortex after peripheral facial palsy. Neurology. 1996;46(2):5001-5001.

9. $\mathrm{Hu} \mathrm{S}, \mathrm{Wu}$ YY, Li CF, et al. Increasing functional connectivity of the anterior cingulate cortex during the course of recovery from Bell's palsy. Neuroreport. 2015;26(1):6-12.

10. Li CF, Yang J, Sun JB, et al. Brain Responses to Acupuncture Are Probably Dependent on the Brain Functional Status. Evid Based Complement Alternat Med. 2013;2013:175278.

11. Kong JA, Ma L, Gollub RL, et al. A pilot study of functional magnetic resonance imaging of the brain during manual and electroacupuncture stimulation of acupuncture point (LI-4 Hegu) in normal subjects reveals differential brain activation between methods. J Altern Complem Med. 2202;8(4):411-419.

12. Douaud G, Mackay C, Andersson J, et al. Schizophrenia delays and alters maturation of the brain in adolescence. Brain : a journal of neurology. 2009;132(9):2437-2448.

13. Xu SB, Huang B, Zhang CY, et al. Effectiveness of strengthened stimulation during acupuncture for the treatment of Bell palsy: a randomized controlled trial. CMAJ. 2013;185(6):473-479.
14. Morecraft RJ, Stilwell-Morecraft KS, Rossing WR. The motor cortex and facial expression: New insights from neuroscience. Neurologist. 2004;10(5):235-249.

15. Valls-Sole J, Montero J. Movement disorders in patients with peripheral facial palsy. Movement Disord. 2003;18(12):1424-1435.

16. Valls-Sole J. Facial palsy, postparalytic facial syndrome, and hemifacial spasm. Movement Disord. 2002;17:S49-S52.

17. Caramia MD, Telera S, Palmieri MG, et al. Ipsilateral motor activation in patients with cerebral gliomas. Neurology. 1998;51(1):196-202.

18. Cattaneo L, Pavesi G. The facial motor system. Neurosci Biobehav R. 2014:38:135-159.

19. Grefkes C, Nowak DA, Wang LE, et al. Modulating cortical connectivity in stroke patients by rTMS assessed with fMRI and dynamic causal modeling. Neuroimage. 2010;50(1):233-242.

20. Squire LR, Stark CEL, Clark RE. The medial temporal lobe. Annu Rev Neurosci. 2004;27:279-306.

21. Buckner RL, Andrews-Hanna JR, Schacter DL. The brain's default network - Anatomy, function, and relevance to disease. Ann Ny Acad Sci. 2008;1124:1-38.

22. Vogt BA. Pain and emotion interactions in subregions of the cingulate gyrus. Nat Rev Neurosci. 2007;6(7):533-544.

23. Cavanna AE. The precuneus and consciousness. CNS Spectr 2007;12(7):545-552.

24. Coulson SE, O'Dwyer TJ, Adams RD, et al. Expression of emotion and quality of life after facial nerve paralysis. Otol Neurotol. 2004;25(6):1014-1019. 